\title{
Epidemiologia Dos Acidentes Com Fraturas Na Infância: O Retrato De Um Município Da Amazônia Brasileira
}

Epidemiology of Accidents With Fracture In Childhood: The Portrait of a Municipality Of The Brazilian Amazon

Epidemiología De Los Accidentes Con Fractura En La Infancia: El Retrato De Un Municipio De La Amazonia Brasileña

Gutembergue Santos de Sousa ${ }^{1}$, Lirian Raquel Bezerra de Sousa ${ }^{2}$, Maria Goreth Silva Ferreira ${ }^{3}$

\section{Resumo}

As lesões traumáticas, na ortopedia e traumatologia, se constituem na mais importante causa de morte em crianças com mais de um ano de idade, em todo o mundo, e na segunda principal causa de hospitalização nos indivíduos com menos de 15 anos de idade. Assim, este estudo objetiva levantar as características epidemiológicas das crianças atendidas com fraturas na internação pediátrica, no Hospital Municipal de Santarém-Pa. Trata-se de um estudo transversal, retrospectivo, realizado através de consulta em prontuários, com população de 183 crianças de 0 a 12 anos, internadas no Hospital Municipal de Santarém-Pa, com

\footnotetext{
${ }^{1}$ Especialista em Auditoria em Serviços de Saúde pela PUC-Go; Especialista em Ortopedia e Traumatologia pela UEPA; Mestre em Saúde na Amazônia pelo NMT/UFPA;

${ }^{2}$ Enfermeira; Especialista em Ortopedia e

Traumatologia (UEPA).

${ }^{3}$ Enfermeira; Doutora em Enfermagem

(EEAN/UFRJ);

Professora Adjunta da Universidade do Estado do

Pará, Campus XII, Santarém-Pará/Brasil
}

quadro de trauma ortopédico, durante o ano de 2013. Evidenciou-se que $69 \%$ dos pacientes eram do sexo masculino, na faixa etária entre 5 a 9 anos $(46,45 \%)$. As crianças residentes em Santarém representaram 69,95\%. As fraturas fechadas representaram $78 \%$, com internação de caráter urgente em 90\% dos casos, tendo o acidente por queda como causa básica em 49,17\%. Nos casos em que a causa básica foi acidentes de trânsito $(9,29 \%)$, estes foram através de motocicletas em 52,94\% dos casos. Diante disto, conclui-se que as crianças internadas possuem características semelhantes às descritas na literatura, sendo predominante as fraturas em crianças do sexo masculino e em idade escolar.

Descritores: Fratura; Criança; Ortopedia; Traumatologia; Epidemiologia.

\section{Abstract}


Traumatic injuries in orthopedics and traumatology are the most important cause of death in children over one year of age worldwide and the second leading cause of hospitalization in individuals under 15 years of age. Thus, this study aims to raise the epidemiological characteristics of children treated with fractures in pediatric hospitalization at the Municipal Hospital of Santarém-Pa. This is a cross-sectional, retrospective study, carried out by means of medical records, with a population of 183 children aged 0 to 12 years admitted to the Municipal Hospital of Santarém-Pa with orthopedic trauma during the year 2013. It was evidenced $69 \%$ of the patients were males, aged between 5 and 9 years $(46.45 \%)$. Children living in Santarém accounted for $69.95 \%$. Closed fractures accounted for $78 \%$, with urgent hospitalization in $90 \%$ of the cases, with the accident due to fall as the underlying cause in $49.17 \%$. In cases where the basic cause were traffic accidents $(9.29 \%)$, these were by motorcycles in $52.94 \%$ of the cases. In view of this, it is concluded that hospitalized children have characteristics similar to those described in the literature, being predominant the fractures in male and school-aged children.

Key words: Fracture; Child; Orthopedics; Traumatology; Epidemiology.

\section{Introdução}

Conhecer o que afeta a população, a partir da multiplicidade de eventos que influem diretamente sobre suas condições de vida e desenvolvimento, é uma forma valiosa para o embasamento de um planejamento mais adequado das ações em saúde, com melhor programação de agendas e prioridades nos vários níveis de gestão $^{(1)}$. Isso pode revelar a demanda por políticas públicas e sociais mais efetivas para que se criem meios de prevenção e controle, ofertando melhorias nas condições de vida e desenvolvimento da população.

As lesões traumáticas, na ortopedia e traumatologia, se constituem na mais importante causa de morte em crianças com mais de um ano de idade, em todo o mundo, e na segunda principal causa de hospitalização nos indivíduos com menos de 15 anos de idade, fato que merece atenção em decorrência da vulnerabilidade a que estão expostas nossas crianças ${ }^{(2)}$.

Dentre as lesões traumáticas comuns na infância, as fraturas ósseas representam uma preocupação por causarem morbidade e seqüelas que podem afetar, especialmente, as articulações e, pelo tempo de recuperação, outras atividades próprias do período de desenvolvimento da criança e pelas repercussões sociais negativas causadas ${ }^{(3)}$. 
Os acidentes e os traumatismos representam um dos maiores desafios para a saúde e o bem-estar das crianças e dos adolescentes. Alguns autores chamam a atenção para o fato de que as fraturas contribuem com uma variação de 10 a $25 \%$ entre todos os traumatismos ocorridos nessa faixa etária ${ }^{(4)}$.

Este estudo teve como objetivo levantar as características epidemiológicas das crianças atendidas na internação pediátrica do Hospital Municipal de Santarém-Pa, HMS, durante o ano de 2013.

\section{Material E Métodos}

Trata-se de um estudo com enfoque quantitativo, tipo descritivo, transversal, retrospectivo, com uma população de 183 crianças de 0 a 12 anos, internadas pelo Serviço de Ortopedia e Traumatologia - SOT, do Hospital Municipal de Santarém, vítimas de trauma ortopédico, durante o ano de 2013. Todas as informações foram obtidas através dos prontuários dos pacientes e do programa de gerenciamento hospitalar - HOSPUB, para, posteriormente, serem repassadas a um formulário adaptado de acordo com as variáveis disponíveis e passarem por um tratamento estatístico.

Tal artigo consiste em um recorte de uma monografia de conclusão de residência multiprofissional, onde objetivou-se traçar o perfil de todos os pacientes internados na enfermaria pediátrica do HMS, pelo SOT.

De acordo com os critérios de exclusão adotados, foram excluídos os prontuários com predominante ausência de informações e dados de pacientes, pacientes referenciados para outras unidades assistenciais ou que vieram a óbito sem passarem pela avaliação do SOT e, ainda, aqueles que deram entrada no período, mas que se encontram em tratamento, não tendo suas informações registradas no banco de dados.

Utilizou-se para a análise dos dados a análise estatísticas simples e o teste de $\mathrm{X}^{2}$. Para tanto, utilizou-se o programa BioEstat 5.3 .

Este estudo não possui conflitos de interesses e foi apreciado e aprovado pelo Comitê de Ética em Pesquisa da Universidade do Estado do Pará, Campus XII - Tapajós, com o número de parecer: 675.541.

\section{Resultados}

Dos pacientes atendidos, 69\% $(\mathrm{n}=126)$ eram do sexo masculino e $31 \% \quad(n=57)$ do sexo feminino. Aplicando o teste do QuiQuadrado, encontramos $X^{2}=26,016$, com $(p)=$ $<0,0001$, com a correção de Yates temos $\mathrm{X}^{2}=$ 25,268 , com $\mathrm{p}=<0,0001$, sendo que os ajustes ou correções não demonstraram diferenças significativas, demonstrando, pelo (p) valor que existe uma elevada significância 
na diferença estatística entre os valores para

01.

cada sexo, conforme representado na tabela

Tabela 01: Distribuição de internação segundo o sexo.

\begin{tabular}{lcccc}
\hline SEXO & $\mathbf{N}$ & $\mathbf{\%}$ & $\mathbf{( p )}$ & (p) Yates \\
\hline Masculino & 126 & 69 & $<0.0001$ & $<0.0001$ \\
Feminino & 57 & 31 & & \\
\hline
\end{tabular}

Fonte: Elaboração própria a partir dos dados coletados.

Com relação à idade, observa-se uma predominância de internação em crianças na faixa etária entre 5 a 9 anos, com $46,45 \%$, seguidos por crianças de 10 a menor de 12 anos, com $34,43 \%$, evidenciando uma maioria de crianças em idade escolar.

Quanto ao tempo de internação, foram encontrados pacientes com duração mínima de 01 dia e máximo de 19 dias, sendo que a maior parte das internações se concentraram na faixa de 1 a 3 dias, em $68,31 \%$ dos casos.
Os principais procedimentos realizados foram redução incruenta de fratura diafisária dos ossos do antebraço, em 20,22\%, e tratamento cirúrgico de fratura ou lesão fisária dos ossos do antebraço, em 19,12\% dos casos. $\mathrm{O}$ quadro 01 resume os principais procedimentos, sendo excluídos todos os procedimentos com ocorrência $n=01$ e $n=$ 02, de modo a facilitar a leitura e compreensão do respectivo quadro.

Quadro 01: Frequência de internação segundo os principais procedimentos realizados.

\begin{tabular}{|l|c|c|}
\hline \multicolumn{1}{|c|}{ Tipo de Procedimento } & N & $\%$ \\
\hline Redução incruenta de fratura diafisária / lesão fisária distal da tíbia com ou sem & 7 & 3,82 \\
\hline Tratamento cirúrgico de fratura / lesão fisária da extremidade proximal do úmero & 4 & 2,18 \\
\hline Tratamento cirúrgico de luxação ou fratura/luxação do cotovelo & & \\
\hline Redução incruenta de fratura / lesão fisária no punho & 4 & 2,18 \\
\hline
\end{tabular}




\begin{tabular}{|c|c|c|}
\hline $\begin{array}{l}\text { Tratamento cirúrgico de fratura da extremidade / metáfise distal dos ossos do } \\
\text { antebraço }\end{array}$ & 5 & 2,73 \\
\hline $\begin{array}{l}\text { Tratamento cirúrgico de fratura de extremidades / metáfise proximal dos ossos do } \\
\text { antebraço }\end{array}$ & 4 & 2,18 \\
\hline Redução incruenta de fratura diafisária dos ossos do antebraço & 37 & 20,22 \\
\hline $\begin{array}{l}\text { Redução incruenta de luxação / fratura-luxação e metacarpo-falangiana / metatarso- } \\
\text { falangiana / inter-falangiana }\end{array}$ & 3 & 1,64 \\
\hline Tratamento cirúrgico de fratura da diáfise do úmero & 16 & 8,74 \\
\hline Tratamento cirúrgico de fratura lesão fisária dos ossos do antebraço & 35 & $19,12 \%$ \\
\hline Amputação / desarticulação de dedo & 6 & 3,28 \\
\hline Tratamento cirúrgico de fratura da diáfise do fêmur & 9 & 4,91 \\
\hline Redução incruenta de fratura diafisária / lesão fisária proximal do fêmur & 7 & 3,82 \\
\hline Tratamento cirúrgico de fratura da diáfise da tíbia & 5 & 2,73 \\
\hline
\end{tabular}

Fonte: Elaboração própria a partir dos dados coletados.

A maioria das internações realizadas foram de crianças residentes em Santarém (69,95\%), seguido por Juruti e Monte Alegre, ambos com 4,92\% dos casos, demonstrando que, se somarmos todos os 13 (treze) municípios que referenciam pacientes de

Tabela 02: Frequência de internação segundo o município de origem.

\section{Procedência dos Atendimentos}

\begin{tabular}{lcc}
\hline Município & N & \% \\
Santarém & 128 & 69,95 \\
Alenquer & 8 & 4,37 \\
\hline
\end{tabular}




\begin{tabular}{ccc}
\hline Itaituba & 3 & 1,64 \\
Juruti & 9 & 4,92 \\
Monte Alegre & 9 & 4,92 \\
Óbidos & 1 & 0,55 \\
Rurópolis & 3 & 1,64 \\
Almeirim & 1 & 0,55 \\
Belterra & 5 & 2,73 \\
Uruará & 2 & 1,09 \\
Curuá & 5 & 2,73 \\
Aveiro & 4 & 2,18 \\
Placas & 1 & 0,55 \\
Prainha & 4 & 2,18
\end{tabular}

Fonte: Elaboração própria a partir dos dados coletados.

$\mathrm{Na}$ tabela 03 podemos observar a frequência de internação segundo os meses do ano. Observa-se maior ocorrência de internação durante os meses de janeiro, com
16,94\% ( $\mathrm{n}=31)$ das internações, setembro, com $12,03 \%(\mathrm{n}=22)$, e outubro, com $11,47 \%$ $(\mathrm{n}=21)$ do total de internações.

Tabela 03: Frequência de internações segundo os meses do ano.

\begin{tabular}{lccc}
\hline Mês & $\mathbf{N}$ & $\mathbf{\%}$ & $\mathbf{( p )}$ \\
\hline Janeiro & 31 & 16,94 & 0004 \\
\hline Fevereiro & 15 & 8,20 & \\
\hline Março & 15 & 8,20 & 7,65 \\
\hline Abril & 14 & 7,10 \\
Maio & 13 & 9,29 \\
\hline Junho & 17 & 4,37 \\
Julho & 8 & 4,37 \\
Agosto & 8 & 12,03
\end{tabular}




\begin{tabular}{lcc} 
Outubro & 21 & 11,47 \\
\hline Novembro & 8 & 4,37 \\
\hline Dezembro & 11 & 6,01
\end{tabular}

Fonte: Elaboração própria a partir dos dados coletados.

Observa-se, também, que não existe uma similaridade entre as proporções de internações durante o ano e, aplicando o teste de $X^{2}$, obtemos $X^{2}=33,59 \operatorname{com}(p)=0,0004$, o que indica existir relevância estatística entre os dados encontrados, não sendo as variações ocorridas durante os meses, em decorrência do acaso.

Quando se avalia as internações pelos dias da semana, observa-se que não existe uma regularidade nesses atendimentos, sendo que o maior número de ocorrência acontece, geralmente, nas quartas-feiras, com 48 ocorrências, e quintas-feiras, com 35 casos. As menores ocorrências foram observadas as segundas-feiras, com 16 casos, e aos sábados, com 18 casos. Ressalta-se que o dia de internação não significa necessariamente o dia de ocorrência do acidente ou do trauma (Figura 01).

Figura 01: Frequência de internações segundo o dia da semana.

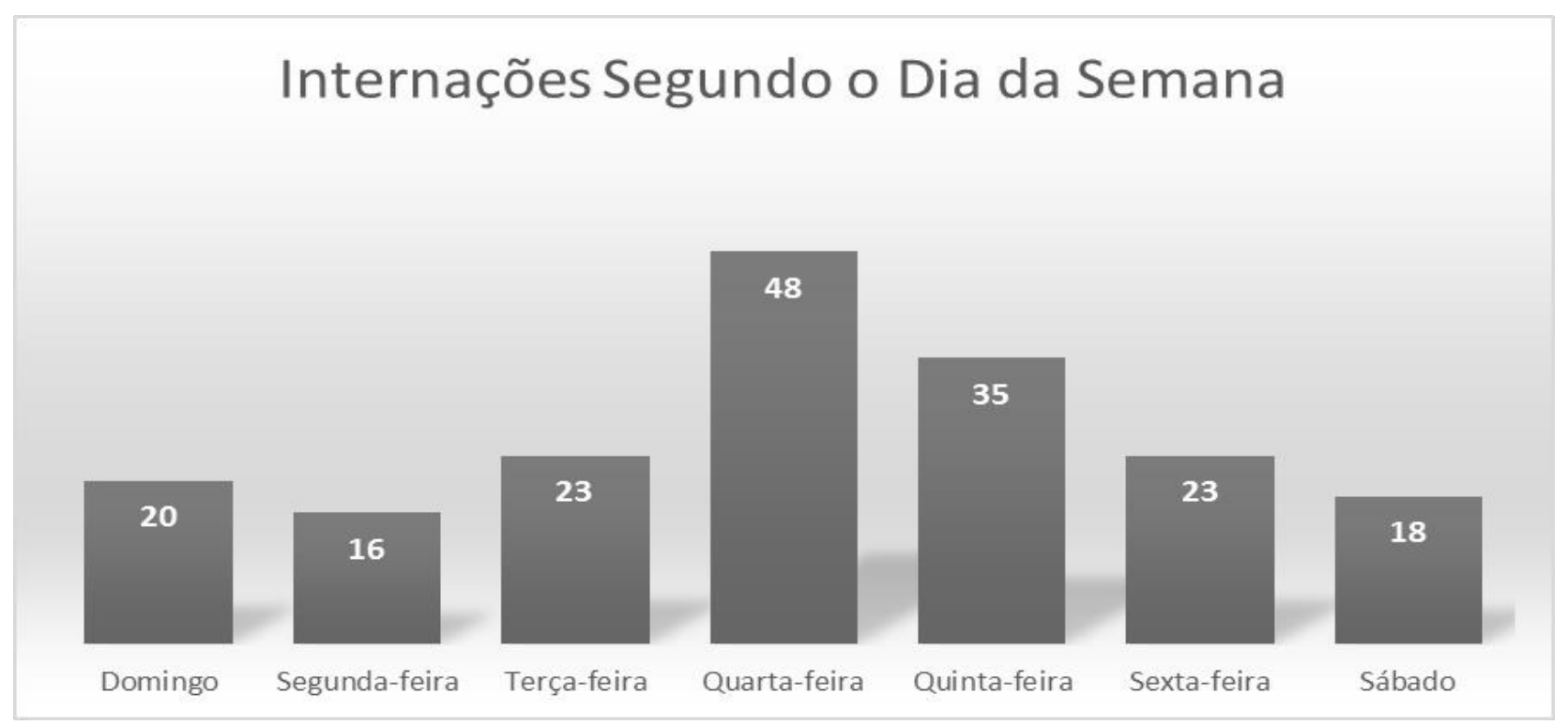

Fonte: Elaboração própria a partir dos dados coletados. 
A maior parte dos atendimentos foi decorrente de fraturas fechadas, com $78 \%$; seguidas por fraturas expostas, com $11 \%$ das internações. Chama a atenção, neste item, a quantidade de prontuários sem informações sobre o tipo de fratura, correspondendo a $6 \%$ do total de internações.

De acordo com o caráter da internação, as internações em casos de urgência representaram $90 \%$ e as de caráter eletivo representaram $10 \%$ dos casos. Este dado demonstra que a maioria dos casos procedentes de Santarém-Pa, ou encaminhados pelos municípios da região de saúde, são para atendimentos imediatos, se constituindo de urgências ou emergências ortopédicas.

Segundo o tipo de acidente avaliado, foi identificado que a maior parte das ocorrências foi em virtude de quedas como causa básica do acidente em 49,17\% dos casos. Os prontuários que não continham esse tipo de informação representaram 36,07 da amostra, evidenciando um grande sub-registro desta informação, dificultando sua análise e inferência para o total de atendimentos.

Observa-se que a frequência de acidentes, segundo o tipo de queda, demonstra uma maior ocorrência de quedas de altura, com 34,43\%, e de árvores (que também se refere a altura mas recebeu uma análise separada), com $18,89 \%$. O percentual de prontuários sem estas informações corresponde a 15,56\%, demonstrando uma grande taxa de prontuários com ausência de informações importantes para análises epidemiológicas.

Nos casos em que a causa básica foi acidentes de trânsito $(9,29 \%)$, estes foram decorrentes, principalmente, de acidentes envolvendo motocicletas, em 52,94\% dos casos.

\section{Discussão}

Percebe-se, pelos dados analisados, uma maior predominância de fraturas no sexo masculino, mantendo uma relação superior a 2:1 entre meninos e meninas. Em um estudo semelhante, onde o autor pesquisou exclusivamente fraturas da diáfise da tíbia, identificou-se também que vários autores evidenciam uma maior frequência de fraturas no sexo masculino. Tal fato, em crianças, pode estar relacionado às próprias atividades lúdicas desempenhadas por cada sexo, sendo as atividades masculinas de maior intensidade e com maior risco para acidentes com trauma ortopédico $^{(5)}$.

$$
\text { Em outro estudo analisado, }
$$
identificou-se, também, um maior acometimento de fraturas em crianças do sexo masculino, numa proporção de, aproximadamente, três meninos para cada menina $(72,5 \%$ para $27,4 \%)$, sendo ambos os dados semelhantes aos encontrados neste trabalho. $\mathrm{O}$ autor ressalta ainda a necessidade 
de direcionamento principal de campanhas educativas para prevenção de acidentes para os adolescentes do sexo masculino ${ }^{(4)}$.

Com relação à idade, observou-se uma predominância de internação em crianças na faixa etária entre 5 a 9 anos, com 46,45\%, seguidas por crianças de 10 a 12 anos, com $34,43 \%$. A maior incidência de fraturas na faixa etária de 5 a 9 anos pode estar relacionado à atividade escolar, a acidentes de trânsito com quedas e à prática esportiva.

Até os sete anos de idade, os traumas mais comuns relatados são decorrentes de acidentes domésticos e escolares; dos 5 aos 10 anos, os atropelamentos são as causas importantes de fratura, enquanto que dos sete aos 14 anos, os acidentes por queda de bicicleta, os esportes (futebol, vôlei, basquete etc), a prática de skate e as chamadas atividades esportivas radicais ${ }^{(5)}$.

Em um estudo, onde realizou-se um levantamento estatístico sobre o trauma ortopédico infanto juvenil, observou-se que na faixa etária escolar as crianças experimentam maior independência e interação com a sociedade, tendendo a se expor mais aos traumas ${ }^{(6)}$.

Em relação ao tempo de permanência em internação hospitalar, observa-se que a maioria das crianças apresentou curto período de internação hospitalar (até 3 dias), sendo alto também o percentual de crianças que tiveram permanência entre 4 e 6 dias $(19,67 \%)$. Tais dados demonstram não existir demora no período de internação, sugerindo um rápido tempo de resposta ao problema apresentado. Evidenciou-se, também, que são poucos os casos que apresentaram período de internação superior a 10 dias, aplicados, geralmente, a casos mais graves e que requerem maior acompanhamento e atenção hospitalar especial.

Em um estudo sobre fratura de fêmur e outros ossos dos membros inferiores, identificou-se que o tempo de permanência em internação hospitalar depende, significativamente, do procedimento realizado e observou, em um contexto geral, uma queda no período de internação hospitalar comparando o ano de 1998 e 2008, e atribuise esta queda a hipótese de melhoria nas condições de promoção da saúde e das políticas de prevenção trabalhadas através de ações intersetoriais de abordagens às causas externas $^{(7)}$.

Ao avaliar os principais procedimentos realizados nas crianças vítimas de fraturas ou patologias ortopédicas, percebemos que mais de $65 \%$ do total se refere a procedimentos realizados nos ossos dos superiores, e, destes, a maior incidência ocorre em ossos do antebraço. Um estudo de fraturas com crianças observou que é maior o acometimento dos membros superiores em relação aos membros inferiores, 
representando $76,08 \%$ das fraturas em membros superiores, e $23,91 \%$ nos membros inferiores, sendo estes dados semelhantes ao encontrado no estudo em questão ${ }^{(5)}$.

A razão de maioria das internações serem provenientes do município de Santarém-Pa, advém do fato de tal município ser pólo regional e possuir a maior quantidade de habitantes entre os municípios analisados. Por ser referência regional, o Hospital Municipal de Santarém recebe pacientes de todos os municípios da região oeste do Pará, através de pactuações de atendimentos e internações especializadas, além da demanda espontânea da população de outros municípios que dão entrada sem o devido processo de regulação.

Quanto ao mês de ocorrência da internação hospitalar, como demonstrado na análise dos dados, observamos que a maior ocorrência se deu nos meses de janeiro e setembro. Na literatura pesquisada não foi encontrada discussão semelhante e que procure identificar o mês com maior ocorrência de internação por trauma ou problemas ortopédicos em crianças. Como a maior ocorrência de fraturas ocorre em idade escolar, esperava-se que a concentração maior de acidentes fosse no período letivo, tendo a escola ou o trajeto como fatores desencadeantes, ou nos meses de férias, devido ao maior tempo dedicado às atividades lúdicas e de lazer.
Neste dado vale ressaltar que foi analisado a frequência de internações distribuídas ao longo dos meses do ano, não representando, diretamente, o mês de ocorrência do trauma ou acidente. Acredita-se que esses dados possam sofrer interferências externas como a liberação de leitos pela central de regulação, estações do ano, número de finais de semana, férias escolares e etc., uma vez que, teoricamente, quanto maior o número de acidentes maior será o número de internações.

Em relação ao dia da semana de ocorrência da internação hospitalar, observase maiores variações nas quartas e quintasfeiras, sendo que nos demais dias as variações não seguem um padrão tão discrepante. Vale ressaltar, aqui, que o dado avaliado foi o dia da semana de ocorrência da internação e não da ocorrência do trauma ortopédico, não sendo possível avaliar se ocorrem mais traumas durante os dias de aulas ou durante os finais de semana. Um estudo semelhante identificou que o horário predominante de atendimento na emergência, ocorre principalmente entre 10 e 16 horas, de terça à quinta-feira. Supondo que a criança seja encaminhada imediatamente ao hospital após a suspeita de fratura, e que, a partir daí, seja providenciada sua internação hospitalar, os dados são semelhantes aos que encontramos em nosso estudo no que se refere ao dia da semana de ocorrência da internação ${ }^{(6)}$. 
$\mathrm{Na}$ comparação entre os dias da semana para fraturas de adultos, um estudo identificou que a ocorrência dos traumas ortopédicos, segundo o dia da semana e o horário do acontecimento, teve maior incidência nas quartas-feiras, sexta- feiras e finais de semana e no intervalo de tempo entre 18 horas e 24 horas $^{(8)}$.

As fraturas fechadas representaram um total de $78 \%$ da amostra, sendo responsável pela maior parte dos atendimentos realizados. Um estudo realizado, principalmente, com crianças e, especificamente, sobre fratura de fêmur, relata que a maior parte dos atendimentos realizados são decorrentes de fraturas fechadas. Em um outro estudo sobre fraturas de úmero em crianças, também identificou-se um maior percentual de ocorrência de fraturas fechadas. Diante de tais dados, observa-se que, frequentemente, ocorre maior incidência de fraturas fechadas em crianças, independentemente da localização anatômica destas fraturas $^{(9)}$.

Ao avaliar o caráter da internação na amostra estudada, observa-se que a maior parte compreende os atendimentos emergenciais, em $90 \%$ da amostra. Por ser um hospital de referência para os demais municípios da região, notou-se que após a estabilização do paciente, o mesmo é encaminhado imediatamente para tratamento ortopédico ou em traumatologia. Observa-se pela literatura que este dado sofre influência de acordo com o perfil do hospital, variando entre atendimentos de urgência e emergência e atendimentos eletivos ${ }^{(10)}$.

Quanto à frequência de internação segundo o tipo de acidente, evidenciou-se uma alta taxa de quedas, representando 49, 17\% da amostra. Chama a atenção, também, nesta análise, o alto índice de subnotificação, representando $36,07 \%$ da amostra, sendo este dado bastante significativo e que requer a adoção de medidas de controle a fim de que sejam geradas informações fidedignas e que retratem o real perfil dos usuários atendidos nesta unidade hospitalar. Em um estudo semelhante, a queda também representou o principal mecanismo de trauma em crianças, sendo o ambiente domiciliar, o principal ambiente de ocorrência desses acidentes, sendo a maioria dos casos representados por quedas de altura (própria altura ou semelhante $)^{(6)}$.

Dentre os acidentes de trânsito observados $(9,29 \%)$, estes foram decorrentes, principalmente, de acidentes envolvendo motocicletas, em 52,94\% dos casos, seguidos pelos acidentes envolvendo bicicletas, em $29,41 \%$. Observa-se uma grande ocorrência de acidentes com bicicletas, provavelmente ocorridos durante atividades lúdicas e de lazer na infância. Tanto no sexo masculino como no feminino, o atropelamento e os acidentes com bicicleta foram as principais causas das 
internações entre os acidentes de trânsito, evidenciados através de uma análise de diversos indicadores em grandes centros urbanos $^{(11)}$.

Encontrou-se uma grande subnotificação de dados essenciais a avaliação em saúde, causando o risco de viés na pesquisa e prejuízos na análise de indicadores institucionais importantes, se fazendo imprescindível uma reflexão sobre a necessidade do preenchimento adequado e completo das informações indispensáveis sobre o estado de saúde do cliente e das condutas adotadas, de modo que o prontuário seja um arquivo detalhado e que retrate, fidedignamente, $\mathrm{o}$ atendimento prestado.

\section{Conclusão}

A partir dos dados coletados e de sua respectiva análise, conclui-se que o perfil das crianças atendidas no serviço de ortopedia e traumatologia, em questão, representa, principalmente, internações em caráter de urgência, de crianças de sexo masculino, na faixa etária de 05 a 09 anos de idade, para tratamento cirúrgico de fratura fechada dos ossos do antebraço, provenientes de Santarém-Pa, com internação média de 01 a 03 dias de duração, ocorridas em sua maioria nas quartas e quintas feiras dos meses de janeiro, setembro e outubro, tendo a queda como principal causa da fratura ou acidente.
Em relação à incidência de fraturas nas crianças e nos adolescentes, temos poucos dados, pois são pequenos os volumes de estudos epidemiológicos a este respeito, em nosso meio. Estudos como este, apresentam dados importantes para a discussão de medidas protetivas às crianças, através de ações de promoção à saúde e prevenção de acidentes que provoquem algum dano à saúde deste grupo etário.

O perfil epidemiológico levantado serve de base para o planejamento institucional do serviço de saúde avaliado, bem como de outros serviços de saúde, no intuito de garantir melhoria e aperfeiçoamento naqueles indicadores considerados de análise crítica. Se fazem necessários novos estudos e estudos mais detalhados sobre a criança vítima de trauma ou afecções ortopédicas, de modo a gerar informações úteis e válidas para que o sistema municipal de saúde possa redirecionar suas ações através da implantação de novos serviços ou estratégias de atuação ou implementação dos serviços já existentes. 


\section{Referências}

1. Pereira MG. Epidemiologia: teoria e prática. 11 ed. Rio de Janeiro: Editora Guanabara; 2007.

2. Irwin Júnior CE, Cataldo MF, Matheny Júnior AP, Peterson L. Health consequences of behaviors: injury as a model. Pediatrics. 1992 [acesso em 24 ago 2014]; 90:798-80. Disponível em:

http://www.ncbi.nlm.nih.gov/pubmed/1437410.

3. Dias GAS, Pontes LS. Perfil epidemiológico de fratura traumática isolada de rádio em crianças. Rev. para. Med. 2013 [acesso 24 ago 2014]; 27(1). Disponível em: http://files.bvs.br/upload/S/01015907/2013/v27n1/a3527.pdf.

4. Guarnieiro R, Godoy Junior RM, Ambrosini Junior E, Guarnieiro JRB, Martins B, Santana PJ et al. Estudo observacional comparativo de fraturas em crianças e adolescentes. Rev. bras. ortop.[online]. 2011; 46(suppl 4): pp. 32-37.

5. Santili C, Gomes CMO, Akkari M, Waisberg G, Braga SR, Lino Junior W et al. Fraturas da diáfise da tíbia em crianças. Acta ortop. bras. [online]. 2010 [acesso 13 out 2014]; 18(1): 44-48. Disponível em: http://www.scielo.br/scielo.php?script=sci_arttext $\underline{\text { \&pid=S1413-78522010000100009. }}$.

6. Lino Júnior $\mathrm{W}$, Segal $\mathrm{AB}$, Carvalho DE, Fregoneze M, Santili C. Análise estatística do trauma ortopédico infanto-juvenil do pronto socorro de ortopedia de uma metrópole tropical. Acta ortop bras. 2005 [12 set 2014]; 13(4): 179$182 . \quad$ Disponível em: www.scielo.br/pdf/aob/v13n4/a05v13n4.pdf.

7. Maciel SSSV, Maciel WV, Lima Neto AJ, Santos FJF, Sobral HV, Sobral LV. Internação hospitalar por fraturas de fêmur e outros ossos dos membros em residentes de Pernambuco. Revista da
AMRIGS. 2012 [acesso em 15 set 2012]; 56 (3): 213-219. Disponível em: http://www.amrigs.com.br/revista/5603/internacao\%20hospitalar.pdf.

8. Itami LT. Adultos com fraturas: das implicações funcionais e cirúrgicas à educação em saúde. Rev Esc Enferm USP. 2009 [acesso 10 set 2014]; 43(Esp 2):1238-43. Disponível em: www.revistas.usp.br/reeusp/article/download/404 $\underline{46 / 43460 .}$.

9. Cunha FM, Figueiredo LA, Coelho LFA, Malheiros DS, Terra DL, Lima CLFA. Fraturas diafisárias de fêmur em crianças e adolescentes. Acta ortop. bras. [online]. 2007 [acesso 13 ago 2014]; 15(2): 80-83. Disponível em: http://www.scielo.br/scielo.php?pid=S141378522007000200004\&script=sci_arttext.

10. Fumo C, Bongiovanni RR, Dobashi ET, Pascarelli L, Righi LCS. Fraturas Supracondilianas de úmero na infância. Arquivos Brasileiros de Ciências da Saúde. 2010 [acesso 09 set 2014]; 35(1):50-56. Disponível em: http://files.bvs.br/upload/S/19832451/2010/v35n1/a013.pdf.

11. Jorge MHPM, Martins CBGA. A criança, o adolescente e o trânsito: algumas reflexões importantes. Rev. Assoc. Med. Bras. [online]. 2013 [acesso 13 ago 2014]; 59(3):199-208. Disponível em: http://www.scielo.br/pdf/ramb/v59n3/v59n3a01.p df.

\section{Participação dos autores:}

SOUSA, LRB trabalhou na concepção teórica, coleta de dados, análise estatística e elaboração e redação final do texto;

SOUSA, GS trabalhou na concepção teórica, elaboração e redação final do texto;

FERREIRA, MGS trabalhou na concepção teórica, elaboração e redação final do texto;

$$
\begin{aligned}
& \text { Recebido: } 31.01 .2017 \\
& \text { Revisado: } 31.01 .2017 \\
& \text { Aprovado: } 31.01 .2017
\end{aligned}
$$

